Kyoto University,

Graduate School of Economics

Research Project Center Discussion Paper Series

Comparative Statics for Oligopoly: A Generalized Result

Naoto Jinji

Discussion Paper No. E-12-011

Research Project Center

Graduate School of Economics

Kyoto University

Yoshida-Honmachi, Sakyo-ku

Kyoto City, 606-8501, Japan

January 2013 


\title{
Comparative Statics for Oligopoly: A Generalized Result*
}

\author{
Naoto Jinji ${ }^{\dagger}$
}

This version: January 23, 2013

\begin{abstract}
We perform comparative statics for a general model of asymmetric oligopoly and derive a concise formula for the response of one firm to a marginal change in its rival's strategic variable, taking into account the responses of all other firms. We obtain the conditions under which the sign of this response coincides with that of the mixed second-order partial derivative of the firm's payoff function. We then propose a distinction between gross and net strategic relationships (i.e., strategic substitute and complement).
\end{abstract}

Keywords: oligopoly; comparative statics; asymmetry; stability conditions.

JEL classification: L13; D43; C62.

\footnotetext{
${ }^{*}$ I thank Keisaku Higashida, Ngo Van Long, Tsuyoshi Toshimitsu, and participants of the 1st HITS-JT seminar on international economy and industry at Aomori Public College for helpful comments and suggestions on earlier versions of the paper. Financial support from the Japan Society for the Promotion of Science under the Grant-in-Aid for Scientific Research (B) No. 23330087 and (C) No. 24530246 is gratefully acknowledged. The usual disclaimer applies.

${ }^{\dagger}$ Faculty of Economics, Kyoto University, Yoshida-honmachi, Sakyo-ku, Kyoto 606-8501, Japan. Phone \& fax: +8175-753-3511. E-mail: jinji \#at-mark\# econ.kyoto-u.ac.jp.
} 


\section{Introduction}

Oligopoly models have been used extensively in many fields of economics, including industrial organization, international trade, public economics, and environmental economics. Comparative statics of oligopolistic equilibria is important to examine the effects of various policies and certain exogenous events. Dixit's (1986) seminal work demonstrated a number of useful properties of comparative statics for oligopoly. However, his analysis addresses only oligopoly with a homogeneous good. Several studies have investigated oligopolistic equilibria's stability conditions (e.g., Hahn, 1962; Seade, 1980; Dixit, 1986; Leahy and Neary, 1997) but have dealt with only symmetric oligopolies or oligopolies for a homogeneous good. To our knowledge, no previous study has identified stability conditions for asymmetric oligopoly with a differentiated good.

Models of asymmetric oligopoly have been applied to many fields. ${ }^{1}$ However, these existing studies introduce asymmetry into oligopolistic models in a specific manner, and no study has analyzed asymmetric oligopoly in a general framework.

For comparative statics, it is worthwhile to generalize to asymmetric oligopoly. For example, suppose you want to know the equilibrium response of one firm (say firm $j$ ) to another firm's (say firm $i$ ) marginal deviation from the initial Nash equilibrium. Because all other firms also respond to firm $i$ 's deviation, in general, you need to take into account the effects of all other firms' equilibrium responses on the response of firm $j$. However, under symmetry, the analysis is very simple as far as the sign of the equilibrium response of firm $j$ is concerned. This is because the sign of firm $j$ 's response to firm $i$ 's deviation, taking into account all other firms' equilibrium response, is always the same as that ignoring all other firms' equilibrium response. Consequently, all you need to know is the sign of the second-order partial derivative of firm $j$ 's payoff function with respect to firm $i$ 's and firm $j$ 's strategic variables. When we

\footnotetext{
${ }^{1}$ See, for example, Ino and Kawamori (2009), Ohkawa et al. (2005), and Zheng et al. (2010) for industrial organization, Collie (1993, 2006), Lahiri and Ono (1997), and Long and Soubeyran (1997) for international trade, Denicolò and Matteuzzi (2000), Lahiri and Ono (1988), and Wang and Zhao (2009) for public economics, and Fujiwara (2011) and Long and Soubeyran (2005) for environmental and natural resource economics.
} 
generalize the model to asymmetric oligopoly with a differentiated good, in contrast, this useful property no longer holds. ${ }^{2}$ Thus, you must investigate more than the sign of the second-order partial derivative of firm $j$ 's payoff function. In this note, we derive a concise formula for the equilibrium response of firm $j$ to a marginal deviation of firm $i$ from the initial Nash equilibrium, taking into account the equilibrium response of all other firms, which has been overlooked by previous studies. We also derive conditions under which the property of symmetric oligopoly passes through to asymmetric oligopoly. Finally, we propose a distinction between gross and net strategic relationship (i.e., strategic substitute and complement) among strategic variables for firms.

Our result has broad application. It can be applied to the analysis of various government policies, such as taxes and subsidies. It is also applicable to certain exogenous shocks such as labor strikes (e.g., Gauder and Salant, 1991) and cost reductions (e.g., Février and Linnemer, 2004) that disturb the firms' production. This note provides a useful tool to studies in many fields of economics that employ oligopoly models.

The rest of this paper proceeds as follows. Section 2 sets up the model and proves the study's main results. Section 3 provides concluding remarks.

\section{Comparative Statics for a General Model of Oligopoly}

Consider an oligopoly model with $n$ firms, where $n \geq 3$ is fixed. ${ }^{3}$ Let $x_{i}$ be the strategic variable for firm $i ; i=1,2, \ldots, n$; and $\pi^{i}\left(x_{i}, \boldsymbol{x}_{-i} ; \boldsymbol{\theta}\right)$ be the payoff for firm $i$, where $\boldsymbol{x}_{-i}$ is a vector of strategic variables for all firms other than firm $i$ and $\boldsymbol{\theta}=\left(\theta_{1}, \ldots, \theta_{n}\right)$ is a vector of parameters. The strategic variable $x_{i}$ could be price, output, or any other decision variable for firms. The parameter $\theta_{i}$ can also be interpreted in many ways. For example, it may represent an exogenous demand or cost shift, a tax/subsidy or other government policy instrument, or a firm's prior-stage choice variable, such as research and development (R\&D), capacity, or advertisement (Dixit, 1986). Alternatively, it may capture exogenous events such

\footnotetext{
${ }^{2}$ The useful property also holds under asymmetric oligopoly if firms produce a homogeneous good.

${ }^{3}$ Because duopoly is easy to handle, we focus on the case of more than three firms.
} 
as a strike or merger (Gaudet and Salant, 1991). The function $\pi^{i}(\cdot)$ is assumed to be twice continuously differentiable. We focus on a one-shot simultaneous-move game.

Assuming firms' Nash behavior, the first-order condition (FOC) for firm $i$ is given by

$$
\pi_{i}^{i} \equiv \frac{\partial \pi^{i}\left(x_{i}, \boldsymbol{x}_{-i} ; \boldsymbol{\theta}\right)}{\partial x_{i}}=0, \quad i=1,2, \ldots, n
$$

which yields firm $i$ 's reaction function as

$$
x_{i}=R^{i}\left(\boldsymbol{x}_{-i} ; \boldsymbol{\theta}\right), \quad i=1,2, \ldots, n .
$$

As usual, $n$ simultaneous equations given by Eq. (2) are solved to obtain Nash equilibria. In the subsequent analysis, we focus on an interior solution.

Totally differentiate Eq. (1) to yield

$$
\boldsymbol{A z}=-\boldsymbol{B} \boldsymbol{y}
$$

where

$$
\boldsymbol{A}=\left(\begin{array}{ccc}
\pi_{11}^{1} & \cdots & \pi_{1 n}^{1} \\
\vdots & \ddots & \vdots \\
\pi_{n 1}^{n} & \cdots & \pi_{n n}^{n}
\end{array}\right), \quad \boldsymbol{z}=\left(\begin{array}{c}
\mathrm{d} x_{1} \\
\vdots \\
\mathrm{d} x_{n}
\end{array}\right), \quad \boldsymbol{B}=\left(\begin{array}{ccc}
\pi_{1 \theta_{1}}^{1} & \cdots & \pi_{1 \theta_{n}}^{1} \\
\vdots & \ddots & \vdots \\
\pi_{n \theta_{1}}^{n} & \cdots & \pi_{n \theta_{n}}^{n}
\end{array}\right), \quad \boldsymbol{y}=\left(\begin{array}{c}
\mathrm{d} \theta_{1} \\
\vdots \\
\mathrm{d} \theta_{n}
\end{array}\right)
$$

where $\pi_{i j}^{i} \equiv \partial^{2} \pi^{i}\left(x_{i}, \boldsymbol{x}_{-i} ; \boldsymbol{\theta}\right) / \partial x_{j} \partial x_{i}$ and $\pi_{i \theta_{j}}^{i} \equiv \partial^{2} \pi^{i}\left(x_{i}, \boldsymbol{x}_{-i} ; \boldsymbol{\theta}\right) / \partial \theta_{j} \partial x_{i}$. We assume that in general $\pi_{i j}^{i}=\pi_{i k}^{i}$ does not hold for $j \neq i$ and $k \neq i, j$. This must be the case if firms produce asymmetrically differentiated goods.

Denote the determinant of $\boldsymbol{A}$ as $\operatorname{det}(\boldsymbol{A})$. Let $\boldsymbol{A}_{i j}$ be the $(n-1) \times(n-1)$ submatrix of $\boldsymbol{A}$ obtained by removing from $\boldsymbol{A}$ its $i$-th row and $j$-th column. Denote the determinant of $\boldsymbol{A}_{i j}$ by $\operatorname{det}\left(\boldsymbol{A}_{i j}\right)$. The $(i, j)$ th cofactor of $\boldsymbol{A}$ is defined as

$$
C_{i j}(\boldsymbol{A})=(-1)^{i+j} \operatorname{det}\left(\boldsymbol{A}_{i j}\right) .
$$

Thus, it holds that

$$
\operatorname{det}(\boldsymbol{A})=\sum_{j=1}^{n} \pi_{k j}^{k} \cdot C_{k j}(\boldsymbol{A})=\sum_{i=1}^{n} \pi_{i k}^{i} \cdot C_{i k}(\boldsymbol{A}) .
$$


Usually, comparative statics serves to analyze the effects of a change in exogenous parameters (such as tax/subsidy) on endogenous variables and payoffs. The effect of a marginal change in $\theta_{i}$ on the payoff for firm $r$ is analyzed by

$$
\begin{aligned}
\frac{\mathrm{d} \pi^{r}}{\mathrm{~d} \theta_{i}} & =\frac{\partial \pi^{r}}{\partial x_{r}} \frac{\mathrm{d} x_{r}}{\mathrm{~d} \theta_{i}}+\frac{\partial \pi^{r}}{\partial \theta_{i}}+\sum_{j \neq r} \frac{\partial \pi^{r}}{\partial x_{j}} \frac{\mathrm{d} x_{j}}{\mathrm{~d} \theta_{i}} \\
& =\frac{\partial \pi^{r}}{\partial \theta_{i}}+\sum_{j \neq r} \frac{\partial \pi^{r}}{\partial x_{j}} \frac{\mathrm{d} x_{j}}{\mathrm{~d} \theta_{i}}
\end{aligned}
$$

where the FOC (Eq. (1)) is used to obtain the second equality. Thus, in addition to the signs of $\partial \pi^{r} / \partial \theta_{i}$ and $\partial \pi^{r} / \partial x_{j}$, we need to know the sign of $\mathrm{d} x_{j} / \mathrm{d} \theta_{i}$ to determine the sign of $\mathrm{d} \pi^{r} / \mathrm{d} \theta_{i}$. In the general model of oligopoly formulated above, we apply Cramer's rule to Eq. (3) to yield

$$
\frac{\mathrm{d} x_{j}}{\mathrm{~d} \theta_{i}}=-\frac{\sum_{k=1}^{n} \pi_{k \theta_{i}}^{k} C_{k j}(\boldsymbol{A})}{\operatorname{det}(\boldsymbol{A})} \quad \text { for } i, j=1, \ldots, n
$$

It is often the case that $\pi_{k \theta_{i}}^{k}=0$ for $k \neq i$. In that case, (6) is simplified to

$$
\frac{\mathrm{d} x_{j}}{\mathrm{~d} \theta_{i}}=-\frac{\pi_{i \theta_{i}}^{i} C_{i j}(\boldsymbol{A})}{\operatorname{det}(\boldsymbol{A})}
$$

In symmetric oligopoly, $\boldsymbol{A}$ and $\boldsymbol{B}$ respectively have the following structure:

$$
\boldsymbol{A}^{s}=\left(\begin{array}{cccc}
\lambda & \rho & \cdots & \rho \\
\rho & \lambda & \cdots & \rho \\
\vdots & \vdots & \ddots & \vdots \\
\rho & \rho & \cdots & \lambda
\end{array}\right), \quad \boldsymbol{B}^{s}=\left(\begin{array}{cccc}
\delta & \eta & \cdots & \eta \\
\eta & \delta & \cdots & \eta \\
\vdots & \vdots & \ddots & \vdots \\
\eta & \eta & \cdots & \delta
\end{array}\right),
$$

where $\lambda \equiv \pi_{i i}^{i}, \rho \equiv \pi_{i j}^{i}, \delta \equiv \pi_{i \theta_{i}}^{i}$, and $\eta \equiv \pi_{i \theta_{j}}^{i}$ for all $i$ and $j \neq i$. In such a case, Eq. (6) takes the following forms:

$$
\begin{aligned}
\frac{\mathrm{d} x_{j}}{\mathrm{~d} \theta_{i}} & =\frac{(\lambda-\rho)^{n-2}(\rho \delta-\lambda \eta)}{\operatorname{det}\left(\boldsymbol{A}^{s}\right)} \\
& =\frac{\rho \delta-\lambda \eta}{(\lambda-\rho)[\lambda+(n-1) \rho]} \quad \text { for } j \neq i, \\
\frac{\mathrm{d} x_{i}}{\mathrm{~d} \theta_{i}} & =-\frac{(\lambda-\rho)^{n-2}[\delta(\lambda+(n-2) \rho)-(n-1) \rho \eta]}{\operatorname{det}\left(\boldsymbol{A}^{s}\right)} \\
& =-\frac{\delta[\lambda+(n-2) \rho]-(n-1) \rho \eta}{(\lambda-\rho)[\lambda+(n-1) \rho]},
\end{aligned}
$$


where $\operatorname{det}\left(\boldsymbol{A}^{s}\right)=(\lambda-\rho)^{n-1}[\lambda+(n-1) \rho]$ is used to derive the second line in each equation.

Suppose now that firm $i$ deviates from the initial Nash equilibrium (because of, for example, an exogenous event). How does this deviation affect each firm's payoff? To answer this question, we need to know how other firms respond to firm i's deviation. Similar to Eq. (5), the effect of a marginal change in $x_{i}$ on firm $r$ 's payoff is given by

$$
\frac{\mathrm{d} \pi^{r}}{\mathrm{~d} x_{i}}=\frac{\partial \pi^{r}}{\partial x_{i}}+\sum_{j \neq i} \frac{\partial \pi^{r}}{\partial x_{j}} \frac{\mathrm{d} x_{j}}{\mathrm{~d} x_{i}}
$$

If $r=i$, then the first term on the right-hand side is equal to zero from the FOC (Eq. (1)). To sign $\mathrm{d} \pi^{r} / \mathrm{d} x_{i}$, we need to know the signs of all of $\mathrm{d} x_{j} / \mathrm{d} x_{i}$ terms. Although Gaudet and Salant (1991) analyze a similar issue, they focus only on the symmetric case. As we discussed in the introduction, under symmetric oligopoly the sign of $\mathrm{d} x_{j} / \mathrm{d} x_{i}$ is always the same as the sign of the partial derivative $\pi_{j i}^{j}$, regardless of whether the equilibrium responses of the other firms are taken into account. In contrast, under asymmetric oligopoly with a differentiated good, this is not necessarily true. We demonstrate this below.

For simplicity, consider that in Eq. (3) $\mathrm{d} \theta_{r}=0$ for all $r=1, \ldots, n$, so that the right-hand side of Eq. (3) becomes an $n \times 1$ column vector $\mathbf{0}^{c}=(0, \ldots, 0)^{\prime}$, where a prime $\left(^{\prime}\right)$ indicates the transpose. Then, firm $j$ 's equilibrium response to firm $i$ 's marginal deviation is formulated in the following lemma:

Lemma 1 Assume that $\operatorname{det}(\boldsymbol{A}) \neq 0$. At Nash equilibrium, it holds that

$$
\frac{\mathrm{d} x_{j}}{\mathrm{~d} x_{i}}=\frac{C_{i j}(\boldsymbol{A})}{C_{i i}(\boldsymbol{A})} \quad \text { for } i=1, \ldots, n, \text { and } j \neq i .
$$

Proof. Denote the $i$-th column of the matrix $\boldsymbol{A}$ by

$$
\boldsymbol{A}_{i}^{c}=\left(\begin{array}{ccc}
\pi_{1 i}^{1} & \cdots & \pi_{n i}^{n}
\end{array}\right)^{\prime} .
$$

Denote an $(n-1) \times 1$ column vector obtained by removing from $\boldsymbol{A}_{i}^{c}$ its $i$-th entry by $\boldsymbol{A}_{i,-i}^{c}$, i.e.,

$$
\boldsymbol{A}_{i,-i}^{c}=\left(\begin{array}{llllll}
\pi_{1 i}^{1} & \cdots & \pi_{(i-1) i}^{i-1} & \pi_{(i+1) i}^{i+1} & \cdots & \pi_{n i}^{n}
\end{array}\right)^{\prime} .
$$


Denote an $(n-1) \times 1$ column vector obtained by removing from $\boldsymbol{z}$ its $i$-th entry and dividing other entries by its $i$-th entry by

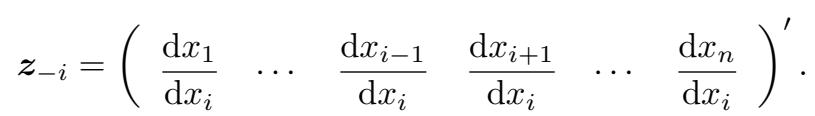

Then, by $\mathrm{d} x_{j} / \mathrm{d} x_{i}, j \neq i$, we analyze the effect of a marginal change in $x_{i}$ on $x_{j}$ at the initial Nash equilibrium. This can be done by solving $(n-1)$ simultaneous equations of

$$
\sum_{k \neq i} \pi_{j k}^{j} \frac{\mathrm{d} x_{k}}{\mathrm{~d} x_{i}}=-\pi_{j i}^{j}, \quad j \neq i
$$

The system of the simultaneous equations can be expressed as

$$
\boldsymbol{A}_{i i} \boldsymbol{z}_{-i}=-\boldsymbol{A}_{i,-i}^{c}
$$

Given that $\boldsymbol{A}_{i i}$ is invertible, this can be solved as

$$
\boldsymbol{z}_{-i}=-\boldsymbol{A}_{i i}^{-1} \boldsymbol{A}_{i,-i}^{c},
$$

where $\boldsymbol{A}_{i i}^{-1}$ is the inverse of $\boldsymbol{A}_{i i}$. Now, construct an $(n-1) \times(n-1)$ matrix $\boldsymbol{C}\left(\boldsymbol{A}_{i i}\right)$ whose $(k, l)$-th entry is $C_{k l}\left(\boldsymbol{A}_{i i}\right)$, which is defined as

$$
C_{k l}\left(\boldsymbol{A}_{i i}\right)= \begin{cases}(-1)^{k+l-1} \operatorname{det}\left(\boldsymbol{A}_{i k, i l}\right) & \text { if } k>i \text { or } l>i \\ (-1)^{k+l} \operatorname{det}\left(\boldsymbol{A}_{i k, i l}\right) & \text { otherwise }\end{cases}
$$

where $\boldsymbol{A}_{i k, \text { il }}$ denotes the $(n-2) \times(n-2)$ submatrix of $\boldsymbol{A}$ that is obtained by removing from $\boldsymbol{A}$ its $i$-th and $k$-th row and $i$-th and $l$-th columns for $k, l \neq i$. Then, the transporse of $\boldsymbol{C}\left(\boldsymbol{A}_{i i}\right)$ is called the adjoint of $\boldsymbol{A}_{\boldsymbol{i} i}$, and is denoted as $\operatorname{Adj}\left(\boldsymbol{A}_{i i}\right)$ :

$$
\operatorname{Adj}\left(\boldsymbol{A}_{i i}\right)=\left(\begin{array}{cccccc}
C_{11}\left(\boldsymbol{A}_{i i}\right) & \cdots & C_{(i-1) 1}\left(\boldsymbol{A}_{i i}\right) & C_{(i+1) 1}\left(\boldsymbol{A}_{i i}\right) & \cdots & C_{n 1}\left(\boldsymbol{A}_{i i}\right) \\
\vdots & \ddots & \vdots & \vdots & \ddots & \vdots \\
C_{1(i-1)}\left(\boldsymbol{A}_{i i}\right) & \cdots & C_{(i-1)(i-1)}\left(\boldsymbol{A}_{i i}\right) & C_{(i+1)(i-1)}\left(\boldsymbol{A}_{i i}\right) & \cdots & C_{n(i-1)}\left(\boldsymbol{A}_{i i}\right) \\
C_{1(i+1)}\left(\boldsymbol{A}_{i i}\right) & \cdots & C_{(i-1)(i+1)}\left(\boldsymbol{A}_{i i}\right) & C_{(i+1)(i+1)}\left(\boldsymbol{A}_{i i}\right) & \cdots & C_{n(i+1)}\left(\boldsymbol{A}_{i i}\right) \\
\vdots & \ddots & \vdots & \vdots & \ddots & \vdots \\
C_{1 n}\left(\boldsymbol{A}_{i i}\right) & \cdots & C_{(i-1) n}\left(\boldsymbol{A}_{i i}\right) & C_{(i+1) n}\left(\boldsymbol{A}_{i i}\right) & \cdots & C_{n n}\left(\boldsymbol{A}_{i i}\right)
\end{array}\right) .
$$


It is known that

$$
\boldsymbol{A}_{i i}^{-1}=\frac{1}{\operatorname{det}\left(\boldsymbol{A}_{i i}\right)} \operatorname{Adj}\left(\boldsymbol{A}_{i i}\right)
$$

holds. Since

$$
\operatorname{det}\left(\boldsymbol{A}_{i i}\right)=C_{i i}(\boldsymbol{A}),
$$

then Eq. (12) can be rewritten as

$$
\boldsymbol{z}_{-i}=-\frac{1}{C_{i i}(\boldsymbol{A})} \operatorname{Adj}\left(\boldsymbol{A}_{i i}\right) \boldsymbol{A}_{i,-i}^{c} .
$$

In the right-hand side of Eq. (13), $-\operatorname{Adj}\left(\boldsymbol{A}_{i i}\right) \boldsymbol{A}_{i,-i}^{c}$ is calculated as

$$
\left(\begin{array}{c}
-\sum_{k \neq i} C_{k 1}\left(\boldsymbol{A}_{i i}\right) \cdot \pi_{k i}^{k} \\
\vdots \\
-\sum_{k \neq i} C_{k n}\left(\boldsymbol{A}_{i i}\right) \cdot \pi_{k i}^{k}
\end{array}\right) .
$$

The $j$-th entry of this column vector is $-\sum_{k \neq i} C_{k j}\left(\boldsymbol{A}_{i i}\right) \cdot \pi_{k i}^{k}$, which is equal to $C_{i j}(\boldsymbol{A})$.

Note that, as shown below, $\boldsymbol{A}_{i i}$ is non-singular under the sufficient condition for stability.

To determine the sign of $\mathrm{d} x_{j} / \mathrm{d} x_{i}$ we need stability conditions. As Seade (1980, Theorem 1$)$, Dixit (1986, p. 117), and Leahy and Neary (1997, Lemma 1) have shown, necessary conditions for stability in a symmetric $n$-firm oligopoly are

$$
\begin{aligned}
\lambda & <0, \\
(-1)^{n}(\lambda-\rho)^{n-1}[\lambda+(n-1) \rho] & >0 .
\end{aligned}
$$

Condition (15) is equivalent to $(-1)^{n} \operatorname{det}\left(\boldsymbol{A}^{s}\right)>0$. Seade (1980, Theorem 1) implies that $\lambda+(n-1) \rho<0$ is necessary for stability. Leahy and Neary (1997, Lemma 1) have shown that a general necessary condition is given by

$$
\lambda<\min \{\rho,-(n-1) \rho\} .
$$

As Seade (1980, Appendix) and Dixit (1986, p. 117) have shown, sufficient condition is obtained by requiring diagonal dominance:

$$
|\lambda|>(n-1)|\rho| .
$$


Then, in a symmetric $n$-firm oligopoly, Leahy and Neary (2009, Lemma 1) have shown that

$$
\frac{\mathrm{d} x_{j}}{\mathrm{~d} x_{i}}=-\frac{\rho}{\lambda+(n-2) \rho} \quad \text { for } \quad j \neq i
$$

This is a special case of Eq. (11). Under the necessary condition for stability $(16), \lambda+(n-2) \rho<0$. Thus, it holds that

$$
\operatorname{sgn}\left(\frac{\mathrm{d} x_{j}}{\mathrm{~d} x_{i}}\right)=\operatorname{sgn}\left(\pi_{j i}^{j}\right)
$$

That is, under symmetry the sign of firm $j$ 's equilibrium response to firm $i$ 's marginal deviation always coincides with the sign of the partial derivative $\pi_{j i}^{j}$. Thus, it does not matter whether the equilibrium responses of all other firms to firm $i$ 's deviation are taken into account.

We then generalize the necessary and sufficient conditions for stability to the case of asymmetric oligopoly. Necessary conditions for stability are

$$
\begin{aligned}
\pi_{i i}^{i} & <0, \quad \text { for } i=1,2, \ldots, n \\
(-1)^{n} \operatorname{det}(\boldsymbol{A}) & >0 .
\end{aligned}
$$

A sufficient condition for stability is given by strictly row diagonal dominance:

$$
\left|\pi_{i i}^{i}\right|>\sum_{j \neq i}\left|\pi_{i j}^{i}\right| \quad \text { for } \quad i=1,2, \ldots, n .
$$

Note that, from the Levy-Desplanques theorem, a strictly diagonally dominant matrix is non-singular (Horn and Johnson, 1985, Theorem 6.1.10). Thus, $\boldsymbol{A}^{-1}$ and $\boldsymbol{A}_{i i}^{-1}$ exist.

Let $\left|M_{k l}\left(\boldsymbol{A}_{i j}\right)\right|$ be the absolute value of the $(k, l)$ th minor in $\boldsymbol{A}_{i j}$, which is the determinant of the $(n-2) \times(n-2)$ submatrix obtained by removing from $\boldsymbol{A}_{i j}$ its $k$-th row and $l$-th column, where $k \neq i$ and $l \neq j .{ }^{4}$ Then, we obtain the following lemma.

Lemma 2 Assume that (22) holds. Then, in $\boldsymbol{A}_{i j}, j \neq i$, it holds that

$$
\left|M_{j i}\left(\boldsymbol{A}_{i j}\right)\right|>\sum_{k \neq i, j}\left|M_{j k}\left(\boldsymbol{A}_{i j}\right)\right|, \quad \text { for } \quad i=1,2, \ldots, n, j=1,2, \ldots, n
$$

\footnotetext{
${ }^{4}$ Note that we count rows and columns by their numbers in the original $\boldsymbol{A}$.
} 
Proof. By definition, $M_{j i}\left(\boldsymbol{A}_{i j}\right)=\operatorname{det}\left(\boldsymbol{A}_{i j, j i}\right)$ and $M_{j k}\left(\boldsymbol{A}_{i j}\right)=\operatorname{det}\left(\boldsymbol{A}_{i j, j k}\right)$, where $j \neq i$ and $k \neq i, j$. Then, since $M_{j i}\left(\boldsymbol{A}_{i j}\right)$ is a principal minor of $\boldsymbol{A}_{i j},(23)$ follows from (22).

In the following lemma, we provide a condition under which the result (19) under symmetric oligopoly also holds under asymmetric oligopoly.

Lemma 3 Assume that stability conditions (20)-(22) hold. Then, in an asymmetric n-firm oligopoly, if

$$
\left|M_{j i}\left(\boldsymbol{A}_{i j}\right)\right|>\sum_{k \neq i, j}\left|\left(\pi_{j k}^{j} / \pi_{j i}^{j}\right)\right|\left|M_{j k}\left(\boldsymbol{A}_{i j}\right)\right|, \quad \text { for } \quad i=1,2, \ldots, n, j=1,2, \ldots, n,
$$

such that $j \neq i$ and $\pi_{j i}^{j} \neq 0$, then it holds that

$$
\operatorname{sgn}\left(\frac{\mathrm{d} x_{j}}{\mathrm{~d} x_{i}}\right)=\operatorname{sgn}\left(\pi_{j i}^{j}\right) \quad \text { for } j \neq i
$$

at Nash equilibrium.

Proof. From Lemma 1, (11) holds. Then, it yields that

$$
\begin{aligned}
\frac{\mathrm{d} x_{j}}{\mathrm{~d} x_{i}} & =\frac{C_{i j}(\boldsymbol{A})}{C_{i i}(\boldsymbol{A})} \\
& =\frac{(-1)^{i+j} \operatorname{det}\left(\boldsymbol{A}_{i j}\right)}{\operatorname{det}\left(\boldsymbol{A}_{i i}\right)} \\
& =\frac{(-1)^{i+j} \sum_{k \neq j} \pi_{j k}^{j} C_{j k}\left(\boldsymbol{A}_{i j}\right)}{\operatorname{det}\left(\boldsymbol{A}_{i i}\right)} \\
& =\frac{(-1)^{i+j} \pi_{j i}^{j}\left(C_{j i}\left(\boldsymbol{A}_{i j}\right)+\sum_{k \neq i, j}\left(\pi_{j k}^{j} / \pi_{j i}^{j}\right) C_{j k}\left(\boldsymbol{A}_{i j}\right)\right)}{\operatorname{det}\left(\boldsymbol{A}_{i i}\right)} .
\end{aligned}
$$

If (24) holds, then $C_{j i}\left(\boldsymbol{A}_{i j}\right)$ dominates the other terms in parentheses in the numerator of (26) and hence it holds that

$$
\operatorname{sgn}\left(\frac{\mathrm{d} x_{j}}{\mathrm{~d} x_{i}}\right)=\operatorname{sgn}\left(\frac{(-1)^{i+j} \pi_{j i}^{j} C_{j i}\left(\boldsymbol{A}_{i j}\right)}{\operatorname{det}\left(\boldsymbol{A}_{i i}\right)}\right) .
$$

Since $C_{j i}\left(\boldsymbol{A}_{i j}\right)=(-1)^{i+j-1} \operatorname{det}\left(\boldsymbol{A}_{i j, i j}\right)$, it can be rewritten as

$$
\operatorname{sgn}\left(\frac{\mathrm{d} x_{j}}{\mathrm{~d} x_{i}}\right)=\operatorname{sgn}\left(\frac{(-1)^{2(i+j)-1} \pi_{j i}^{j} \operatorname{det}\left(\boldsymbol{A}_{i j, i j}\right)}{\operatorname{det}\left(\boldsymbol{A}_{i i}\right)}\right) \text {. }
$$

Since from (20) and (21) it holds that $(-1)^{n-1} \operatorname{det}\left(\boldsymbol{A}_{i i}\right)>0$ and $(-1)^{n-2} \operatorname{det}\left(\boldsymbol{A}_{i j, i j}\right)>0$,

$$
\operatorname{det}\left(\boldsymbol{A}_{i j, i j}\right) / \operatorname{det}\left(\boldsymbol{A}_{i i}\right)<0
$$


always holds. Thus, (25) follows from (27).

Note that if $\pi_{j i}^{j}=\pi_{j k}^{j}$ for all $k \neq i, j$, then (24) follows from (23). Thus, (25) holds under stability conditions (20)-(22). However, in a generalized case, stability conditions (20)-(22) alone do not guarantee that (25) holds. An additional condition (24) must be satisfied for (25) to be true.

As is well known from Bulow et al.'s (1985) terminology, $x_{j}$ is a strategic substitute (resp. strategic complement) to $x_{i}$ if $\pi_{i j}^{j}<0$ (resp. $\left.\pi_{i j}^{j}>0\right)$. However, this definition reflects only the direct relationship between $x_{j}$ and $x_{i}$. As we demonstrated in Lemma 3 , the sign of $\mathrm{d} x_{j} / \mathrm{d} x_{i}$ is not necessarily the same as the sign of $\pi_{i j}^{j}$ in the general model of asymmetric oligopoly. Therefore, it is worthwhile to distinguish between gross and net strategic relationships. We call the strategic relationship determined by the sign of $\pi_{i j}^{j}$ gross strategic substitute and gross strategic complement. In contrast, the strategic relationship determined by the sign of $\mathrm{d} x_{j} / \mathrm{d} x_{i}$ can be called net strategic substitute and net strategic complement. As is evident from the analysis in this note, the net and gross strategic relationships always coincide with each other under symmetric oligopoly and under oligopoly with a homogeneous product. However, under asymmetric oligopoly with a differentiated good, $x_{j}$ could be a gross strategic substitute to $x_{i}$ but a net strategic complement to $x_{i}$. For policy makers who implement a policy before firms' actions, the net strategic relationship is more important for determining the optimal policy.

Lemma 3 implies that under condition (25) the sign of firm $j$ 's equilibrium response to firm $i$ 's marginal deviation, taking into account the responses of all other firms, is the same as the sign of the partial derivative of firm $j$ 's reaction function with respect to $x_{i} .{ }^{5}$ Let $x_{j}=R^{j}\left(\boldsymbol{x}_{-j} ; \boldsymbol{\theta}\right)$ be firm $j$ 's reaction function obtained from firm $j$ 's FOC (Eq. (1)). Then, it yields that

$$
\frac{\mathrm{d} x_{j}}{\mathrm{~d} x_{i}}=\frac{\partial R^{j}}{\partial x_{i}}+\sum_{k \neq i, j} \frac{\partial R^{j}}{\partial x_{k}} \frac{\mathrm{d} x_{k}}{\mathrm{~d} x_{i}}
$$

Condition (24) ensures that in (28) the first term on the right-hand side dominates the sum of all other terms on the right-hand side.

\footnotetext{
${ }^{5}$ I thank Ngo Van Long for suggesting this interpretation.
} 
As is well known, (25) always holds if $n=2$ because in (11) $C_{i j}(\boldsymbol{A})=-\pi_{j i}^{j}$ and $C_{i i}(\boldsymbol{A})=\pi_{j j}^{j}$, where $\pi_{j j}^{j}<0$ from the stability condition.

\section{Concluding Remarks}

In this note, we performed comparative statics in a general framework of oligopoly. It can be applied to various types of competition among firms in oligopolistic markets, including standard price and quantity competition. The results obtained by previous studies such as Seade (1980), Dixit (1986), and Leahy and Neary (1997) are included as special cases of those in this note. Furthermore, by imposing specific assumptions on the structure of the model, this studie's formulas of comparative statics can apply to a number of uninvestigated issues. We encourage such applications.

\section{References}

[1] J.I. Bulow, J.D. Geanakoplos, P.D. Klemperer, Multimarket oligopoly: strategic substitutes and complements, J. Polit. Econ. 93 (1985) 488-511.

[2] D. Collie, Strategic trade policy under asymmetric oligopoly, Eur. J. Polit. Econ. 9 (1993) 275-80.

[3] D. Collie, Tariffs and subsidies under asymmetric oligopoly: ad valorem versus specific instruments, Manch. Sch. 74 (2006) 314-33.

[4] V. Denicolò, M. Matteuzzi, Specific and ad valorem taxation in asymmetric Cournot oligopolies, Int. Tax Public Finan. 7 (2000) 335-42.

[5] A. Dixit, Comparative statics for oligopoly, Int. Econ. Rev. 27 (1986) 107-22.

[6] P. Février, L. Linnemer, Idiosyncratic shocks in an asymmetric Cournot oligopoly, Int. J. Ind. Org. $22(2004) 835-48$. 
[7] K. Fujiwara, Losses from competition in a dynamic game model of a renewable resource oligopoly, Resour. Energ. Econ. 33 (2011) 1-11.

[8] G. Gaudet, S.W. Salant, Increasing the profits of a subset of firms in oligopoly models with strategic substitutes, Amer. Econ. Rev. 81 (1991) 658-65.

[9] F.H. Hahn, The stability of the Cournot oligopoly solution, Rev. Econ. Stud. 29 (1962) 329-31.

[10] R.A. Horn, C.R. Johnson, Matrix Analysis, Cambridge: Cambridge University Press, 1985.

[11] H. Ino, T. Kawamori, Oligopoly with a large number of competitors: asymmetric limit result, Econ. Theory 39 (2009) 331-52.

[12] S. Lahiri, Y. Ono, Helping minor firms reduces welfare, Econ. J. 98 (1988) 1199-202.

[13] S. Lahiri, Y. Ono, Asymmetric oligopoly, international trade, and welfare: a synthesis, J. Econ. 65 (1997) 291-310.

[14] D. Leahy, J.P. Neary, Public policy towards R\&D in oligopolistic industries, Amer. Econ. Rev. 87 (1997) 642-62.

[15] D. Leahy, J.P. Neary, Multilateral subsidy games, Econ. Theory 41 (2009) 41-66.

[16] N.V. Long, A. Soubeyran, Cost heterogeneity, industry concentration and strategic trade policies, J. Int. Econ. 43 (1997) 207-20.

[17] N.V. Long, A. Soubeyran, Selective penalization of polluters: an inf-convolution approach, Econ. Theory 25 (2005) 421-54.

[18] T. Ohkawa, M. Okamura, N. Nakanishi, K. Kiyono, The market selects the wrong firms in the long run, Int. Econ. Rev. 46 (2005) 1143-65.

[19] J. Seade, The stability of Cournot revisited, J. Econ. Theory 23 (1980) 15-27. 
[20] X.H. Wang, J. Zhao, On the efficiency of indirect taxes in differentiated oligopolies with asymmetric costs, J. Econ. 96 (2009) 223-39.

[21] Y. Zheng, T. Bar, H.M. Kauser, Generic advertising in an asymmetric Cournot oligopoly, Am. J. Agr. Econ. 92 (2010) 740-54. 